\title{
Realizing Persistent-Spin-Helix Lasing in the Regime of Rashba-Dresselhaus Spin-Orbit Coupling in a Dye-Filled Liquid-Crystal Optical Microcavity
}

\author{
Marcin Muszyński®, ${ }^{1}$ Mateusz Królø, ${ }^{1}$ Katarzyna Rechcińska®, ${ }^{1}$ Przemysław Oliwa, ${ }^{1}$ \\ Mateusz Kędziora $\odot,{ }^{1}$ Karolina Łempicka-Mirek, ${ }^{1}$ Rafał Mazur $\odot,{ }^{2}$ Przemysław Morawiak $\odot,{ }^{2}$ \\ Wiktor Piecek®, ${ }^{2}$ Przemysław Kula $\odot,{ }^{3}$ Pavlos G. Lagoudakis $\odot,{ }^{4,5}$ Barbara Piętka $\odot,{ }^{1}$ \\ and Jacek Szczytko ${ }^{1, * *}$ \\ ${ }^{1}$ Institute of Experimental Physics, Faculty of Physics, University of Warsaw, 02-093 Warsaw, Poland \\ ${ }^{2}$ Institute of Applied Physics, Military University of Technology, 00-908 Warsaw, Poland \\ ${ }^{3}$ Institute of Chemistry, Military University of Technology, 00-908 Warsaw, Poland \\ ${ }^{4}$ Skolkovo Institute of Science and Technology, Bolshoy Boulevard 30, Bld. 1, Moscow 121205, Russia \\ ${ }^{5}$ Department of Physics and Astronomy, University of Southampton, Southampton SO17 1BJ, United Kingdom
}

(Received 29 September 2021; revised 15 December 2021; accepted 20 December 2021; published 28 January 2022)

In the presence of Rashba-Dresselhaus coupling, strong spin-orbit interactions in liquid-crystal optical cavities result in a distinctive spin-split entangled dispersion. Spin coherence between such modes gives rise to an optically persistent spin helix. In this paper, we introduce optical gain in such a system, by dispersing a molecular dye in a liquid-crystal microcavity, and demonstrate an optically persistent spinhelix lasing in the Rashba-Dresselhaus regime.

DOI: 10.1103/PhysRevApplied.17.014041

\section{INTRODUCTION}

The engineering of the dispersion of photons in microstructured optical systems brings about a plethora of new applications in optoelectronics ranging from information transfer and processing to quantum optics and beyond [1]. Furthermore, it enables the emulation of complex solid-state systems through the realization of synthetic Hamiltonians that can be derived merely from the propagation of electromagnetic waves in appropriately designed photonic microstructures. A compelling example is the photonic analog of spin-orbit interactions in electronic systems, where the pseudospin of a photon mimics the spin of an electron [2]. The ability to engineer artificial gauge fields for photons [3] has led to rapid advances in topological photonics $[4,5]$ and the realization of photonic spin-orbit coupling (SOC) Hamiltonians [6-8] following advancements in cold atoms [9-11] and solids [12-14].

Liquid-crystal (LC) birefringent cavities allow for a wide range of tunability of energy and polarization of confined photonic modes [2,15]. In particular, if two photonic modes of orthogonal polarization and different parity are

*Jacek.Szczytko@fuw.edu.pl

Published by the American Physical Society under the terms of the Creative Commons Attribution 4.0 International license. Further distribution of this work must maintain attribution to the author(s) and the published article's title, journal citation, and DOI. tuned to the resonance, a characteristic scheme of spinpolarized dispersion is formed [2], which in solid-state physics is called the Rashba-Dresselhaus (RD) spin-orbit coupling [16]. The characteristic feature of equal Rashba and Dresselhaus terms is the appearance of a long-range spin texture, the so-called persistent spin helix [17,18], recently also observed in photonic systems [19]. Although such tunable cavities can be easily integrated with various organic [20] and inorganic emitters [21,22] and provide an excellent platform for photonic and optoelectronic devices, lasing in the $\mathrm{RD}$ regime has remained elusive. Here, we combine the recent advances in integrated photonic microcavities filled with a liquid crystal of high birefringence [23] and the tremendous progress in the development of high-efficiency organic semiconductor dyes [24] that can be embedded in liquid-crystal lasers [25], to demonstrate a broadly tunable laser that allows us to reach optical persistent spin-helix lasing.

\section{DYE-DOPED LIQUID-CRYSTAL MICROCAVITIES}

A schematic of our device is shown in Fig. 1(a). The cavity is composed of two $\mathrm{SiO}_{2} / \mathrm{TiO}_{2}$ distributed Bragg reflectors (DBRs) with maximum reflectance at $\lambda=$ $550 \mathrm{~nm}(2.25 \mathrm{eV})$. The two DBRs are joined together at the edges of the sample with a glue containing silica spacers to obtain a distance between the DBRs varying between $2 \mu \mathrm{m}$ and $3 \mu \mathrm{m}$, depending on the position on the sample. 


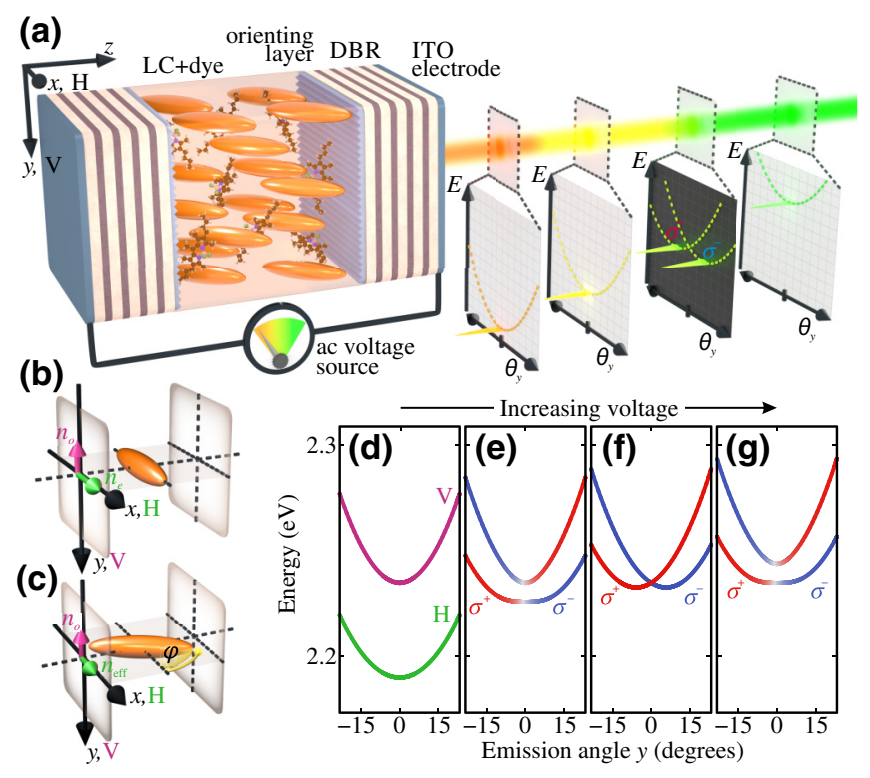

FIG. 1. (a) The scheme of the optical microcavity filled with liquid crystal and P580 dye (dye-doped LCMC). Upon pulsed excitation, lasing action occurs at the lowest energy $E$ of the cavity-modes dispersion relation, which depends on the amplitude of the ac voltage applied to the structure. (b) For initial orientation of the $\mathrm{LC}$, the effective refractive index for vertically (V) [horizontally $(\mathrm{H})$ ] polarized light is equal to the ordinary $n_{o}$ (extraordinary $n_{e}$ ) refractive index. (c) With a voltage applied to the ITO electrodes, the LC molecules rotate within the $x-z$ plane, which decreases the effective refractive index $n_{\text {eff }}$ for horizontally polarized light. (d)-(g) The energies of the cavity modes with increasing electric bias. When two modes of different parity are close to degenerate (e)-(g), they are coupled with the RashbaDresselhaus term, resulting in circular polarization of the bands marked as red for $\sigma^{+}$and blue for $\sigma^{-}$.

This space is filled with a nematic liquid crystal, which acts as an optically uniaxial medium with ordinary $\left(n_{o}\right)$ and extraordinary $\left(n_{e}\right)$ refractive indexes with high birefingence $\left(\Delta n=n_{e}-n_{o}=0.41\right)$. The initial orientation of the LC molecules is determined by the structurization of the polymer-orienting layers present on top of the DBRs and the optically long axis of the LC (the director) is oriented along the $x$ axis, as shown in Fig. 1(b). In such an orientation, the effective refractive index of light polarized linearly along the $y$ axis (vertical, $V$ ) is equal to the ordinary refractive index $\left(n_{o}\right)$. For perpendicular linear polarization along the $x$ axis (horizontal, $\mathrm{H}$ ), the effective refractive index is higher and is equal to $n_{e}$. As the effective refractive indexes differ, the cavity modes in both linear polarizations are separated in energy, which is schematically presented in a band diagram in Fig. 1(d) (green for the $\mathrm{H}$-polarized mode and purple for the V-polarized mode).

The use of transparent electrodes made of indium tin oxide (ITO) allows us to control the direction of the optical anisotropy within the $x-z$ plane, as presented in Fig. 1(c).
With the application of an external electric field, the effective refractive index for the V-polarized light remains constant but it decreases for the H-polarized light. As a result, the V-polarized mode energy remains constant, while the energy of the H-polarized mode increases with the applied electric field.

When horizontal and vertical modes with different parity are close to degeneracy, a Rasha-Dresselhaus spin-orbit coupling interaction appears [2]. The dispersion relation can then be described by the effective Hamiltonian, written in the basis of the photon circular polarizations:

$$
\hat{H}=\frac{\hbar^{2} \vec{k}^{2}}{2 m}-2 \alpha k_{y} \hat{\sigma}_{z}+\frac{1}{2}\left(\Delta E_{\mathrm{HV}}\right) \hat{\sigma}_{x}
$$

where $\vec{k}=\left(k_{x}, k_{y}\right)$ is the cavity in-plane momentum, $m$ is the effective mass of the cavity photon, $\alpha$ is the RashbaDresselhaus coupling coefficient, $\hat{\sigma}_{x}\left(\hat{\sigma}_{z}\right)$ is the first (third) Pauli matrix, and $\Delta E_{\mathrm{HV}}$ corresponds to the energy difference between uncoupled $\mathrm{H}$ - and V-polarized modes. The resulting dispersion relations given by Eq. (1) are presented in Figs. 1(e)-1(g). Apart from the change in the dispersion relation due to Rashba-Dresslehaus spin-orbitcoupling, the polarization of the bands changes to circular, with $\sigma^{+}\left(\sigma^{-}\right)$circular polarizations marked with red (blue) colors.

By doping the cavity with a pyrromethene 580 (P580) laser dye [26,27], the spontaneous emission becomes filtered and transformed by cavity modes. Additionally, the dye acts as a gain medium inside the cavity, making it possible to obtain a laser action. The effect of tuning the emission energy with the applied voltage is conceptually presented in Fig. 1(a).

\section{EXPERIMENTAL RESULTS WITH DISCUSSION}

The optical measurements are performed at room temperature. All data are acquired in a single-shot on-demand experiment. The microcavity is excited by a $Q$-switched diode-pumped laser (532-nm center wavelength, 2-ns pulse duration). The microscope objective with numerical aperture NA $=0.75$ is used for both the excitation and collection of emitted light. The size of the pump spot has a diameter of around $5 \mu \mathrm{m}$. A quarter-wave plate, half-wave plate, and linear polarizer setup are used to detect light in the selected polarization. To tune the cavity modes, we use an external voltage of a square waveform of frequency $1 \mathrm{kHz}$.

Figure 2 shows the lasing effect in dye-doped liquidcrystal microcavity (LCMC). Figures 2(a)-2(c) show angle-resolved photoluminescence spectra close to the Rashba-Dresslehaus regime for increasing energies of the excitation pulse. In Fig. 2(a), the emission spectrum 
obtained below the lasing threshold reveals the dispersion relation of the cavity modes. The solutions of the Rashba-Dresselhaus Hamiltonian given by Eq. (1) are fitted to the experimental results for the two upper and two lower modes independently and are plotted on maps with purple and green dashed lines for horizontal and vertical modes, respectively. Figure 2(b) presents the measurement obtained just above the lasing threshold. At the bottom of the horizontal-mode parabola, enhancement and both spectral and angular narrowing of the light emission take place. Figure 2(c) illustrates the situation well above the threshold, when laser light dominates the entire map. The emission spectra at a $0^{\circ}$ angle for different pulse energies are plotted in Fig. 2(d). The measured spectra are fitted with a Lorentzian line shape and the extracted fitting parameters are shown in Fig. 2(e). The emission intensity (red dots) initially increases linearly with the excitation power, following the black line fitted to the data points below the lasing threshold. For a pulse-energy density of $3.21 \mathrm{~J} / \mathrm{cm}^{2}$ and above, a rapid nonlinear increase in the emission intensity is observed with a concurrent line-width narrowing (blue squares), indicative of a lasing threshold. At the lasing threshold, the emission wavelength exhibits a red shift, followed by a blue shift at higher pumping-pulse energies. We note here that in noncrystalline organic microcavities, in which excitons are localized on individual chromophores or molecules, wavelength blue shifts of the nonlinear emission with increasing excitation density do not indicate the presence of strong coupling [28]. The localized nature of excitons precludes repulsive exchange interactions and the associated blue shifts that are observed with increasing polariton density in crystalline semiconductors. It has recently been shown that even in strongly coupled dye-filled microcavities, the observed blue shift at the condensation threshold is predominantly driven by a renormalization of the intracavity refractive index and to a lesser extent by a quenching of the Rabi splitting [28]. It is therefore essential to dissociate oneself from claims of strong coupling with nonlinear emission blue shifts at the lasing threshold, unless a thorough characterization is performed that can preclude mechanisms that are not associated with strong coupling [28]. Claims of strong coupling should include spectroscopic evidence of anticrossing or adequate differentiation with nonstrongly coupled reference samples $[29,30]$. In this study, we do not identify any evidence of strong coupling and the observed emission wavelength shifts are attributed to excitation-density-induced changes of the intracavity refractive index, including heating.

Since lasing takes place in the horizontal modepolarized in the plane of the rotation of the LC molecules - it provides an opportunity to tune the spectral position of the laser line with the applied external voltage. Figure 3(a)-3(c) shows three normalized angle-resolved emission spectra above the lasing threshold for different applied voltages. The lasing line follows the energy of the

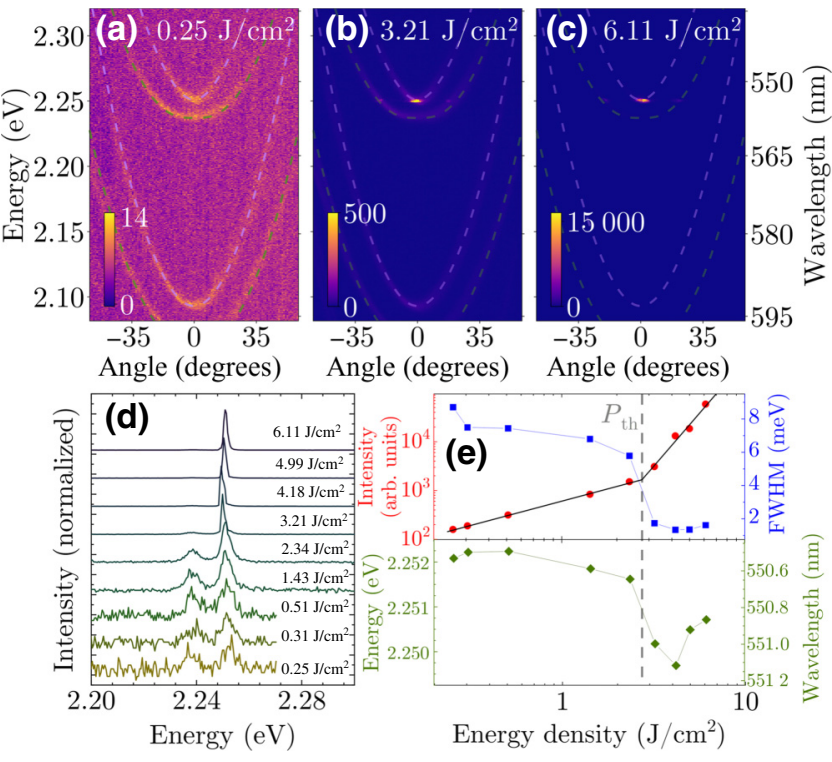

FIG. 2. Lasing in dye-doped LCMC. The experimental emission spectra (a) below and (b),(c) above the lasing threshold. The purple and green dashed lines correspond to the RashbaDresselhaus Hamiltonian (1). (d) Normalized emission spectra obtained for an angle close to $0^{\circ}$ for different pulse energies. (e) The emission intensity (red dots), line width (blue squares), and peak position (green diamonds) as a function of the energy of the excitation pulse. The black lines are the linear fits to the intensity parameters below (first five points) and above (last four points) the lasing threshold.

bottom of the parabola of the horizontal mode. The peak position is shown as a function of the external voltage in Fig. 3(d). Two series of measurements for increasing voltage are performed for a pulse-energy density of $2.34 \mathrm{~J} / \mathrm{cm}^{2}$ (orange dots) and $3.21 \mathrm{~J} / \mathrm{cm}^{2}$ (purple diamonds), both above the lasing threshold. The measurements are taken at different spots on the sample than in Fig. 2; hence the lasing threshold is lower. One can see that the tuning effect is repeatable, as the emission energy in both of the series is almost the same at a given voltage and for this position on the sample the available range of peak positions spans approximately $40 \mathrm{~nm}$. This range is limited by dye gain bandwidth [31] and the sample thickness. The second effect is evident for the voltages where the peak-position wavelength is above $580 \mathrm{~nm}$. In this case, two subsequent horizontal modes begin to compete for the population inversion.

An interesting effect occurs at the mode degeneracy in the regime of optical spin-orbit $\mathrm{RD}$ coupling, with the characteristic dispersion relation schematically plotted in Fig. 4(a). In this case, the lasing takes place simultaneously from the bottoms of two off-centered spin-polarized coupled valleys, as shown in the angle-resolved emission spectrum in Fig. 4(b). Since both valleys are circularly polarized with opposite helicities to each other [2], the laser light also inherits the state of their circular 

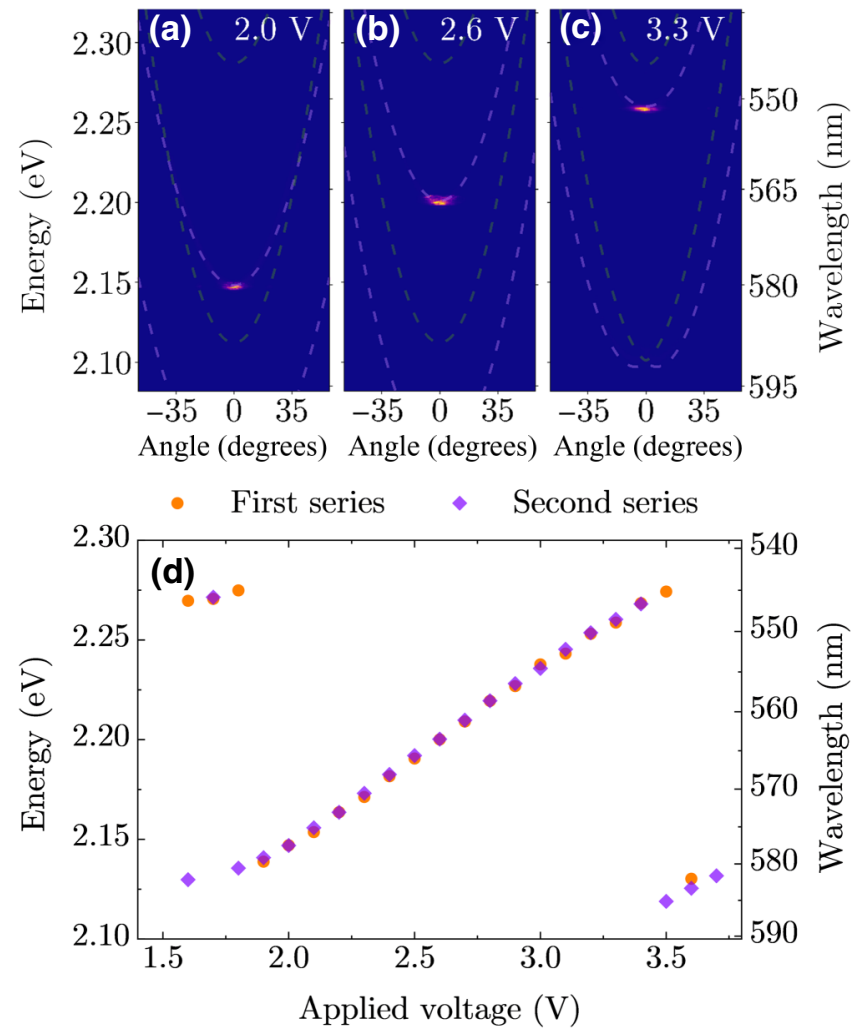

FIG. 3. The tunable laser wavelength. (a)-(c) The normalized experimental emission spectra measured for three different applied voltages above the lasing threshold. (d) The position of the main emission peak versus the applied voltage. The first series (orange dots) and the second series (purple diamonds) are measured for pulse-energy densities of $2.34 \mathrm{~J} / \mathrm{cm}^{2}$ and $3.21 \mathrm{~J} / \mathrm{cm}^{2}$, respectively.

polarization. This effect is evidenced in Fig. 4(c), which presents the difference in the emission intensities detected in $\sigma^{+}$(red) and $\sigma^{-}$(blue) polarizations (the unnormalized $S_{3}$ parameter). Figures 4(d) and 4(e) present corresponding results resolved along both axes of the emission angles but without spectral resolution. In Fig. 4(d), which presents the total emission intensity, one can see two spots spaced apart along the $y$ axis. As expected, both spots are strongly circularly polarized with opposite signs, as is shown in Fig. 4(e), which presents the $S_{3}$ parameter of the emitted light. The separation between the two spots of $\pm 8^{\circ}$ corresponds to $Q=3.1 \mu \mathrm{m}^{-1}$ in momentum space, as the in-plane wave vector is dependent on the emission angle $\theta$ through the formula $k_{\|}=(E / \hbar c) \sin \theta$. The rectangular shape of the maps in Figs. 4(d) and 4(e) is related to the spectrometer slit aperture.

The light emitted in RD conditions can be considered as a pair of beams of circular polarizations with equal intensities given by basis vectors of polarization subspace $\left\{\vec{\sigma}_{-}, \vec{\sigma}_{+}\right\}$and momentum subspace $\{-\vec{Q},+\vec{Q}\}$ [19]. In the far field, this means that the determination of the direction of the light propagation unambiguously also determines its
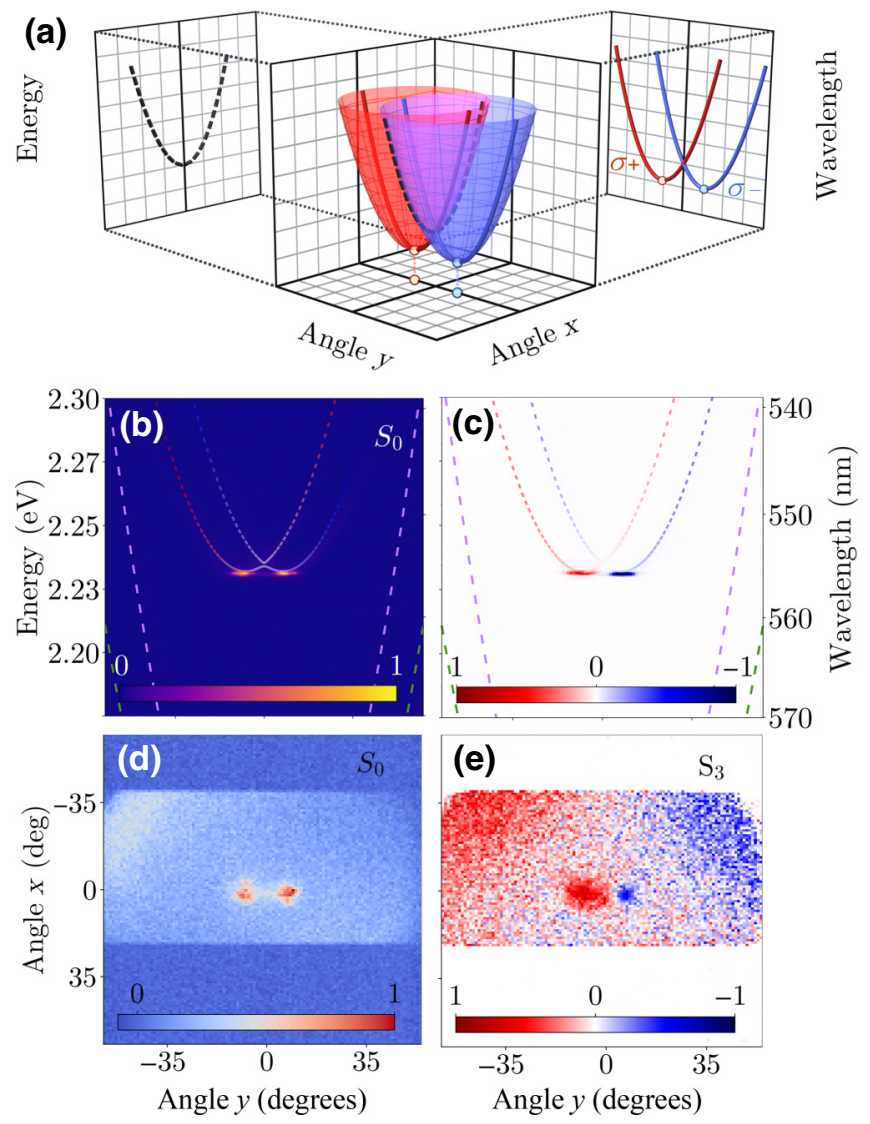

FIG. 4. Lasing in Rashba-Dresselhaus resonance. (a) The dispersion relation in both directions of the incidence angles. (b) Angle-resolved emission spectra. (c) The difference between the emission intensities in $\sigma^{+}$(red) and $\sigma^{-}$(blue) polarization. (d),(e) The momentum-space imaging for (d) the total emission intensity and (e) the degree of circular polarization (the $S_{3}$ parameter).

polarization and vice versa. In other words, the emission in Fig. 4 is separable into pairs $\left\{-\vec{Q}, \vec{\sigma}_{+}\right\}$and $\left\{+\vec{Q}, \vec{\sigma}_{-}\right\}$. In the near field, however, the coherent emission from both dispersion spin valleys leads to the interference pattern of the linearly polarized persistent spin helix (PSH) [19]. Such interference requires emission in both circular polarizations, so it is given by an inseparable state of light mixing all basis vectors in momentum and polarization degrees of freedom. The electric field at the surface of the cavity exactly at the $\mathrm{RD}$ resonance conditions is represented by the components of the normalized Stokes vectors [19]:

$$
\begin{aligned}
& S_{1}(\vec{r})=\cos (\vec{Q} \cdot \vec{r}+2 \Theta), \\
& S_{2}(\vec{r})=-\sin (\vec{Q} \cdot \vec{r}+2 \Theta), \\
& S_{3}(\vec{r})=0,
\end{aligned}
$$

where $\Theta$ is a variable that uniquely determines the location of the linear polarization interference minima and maxima in the PSH. 


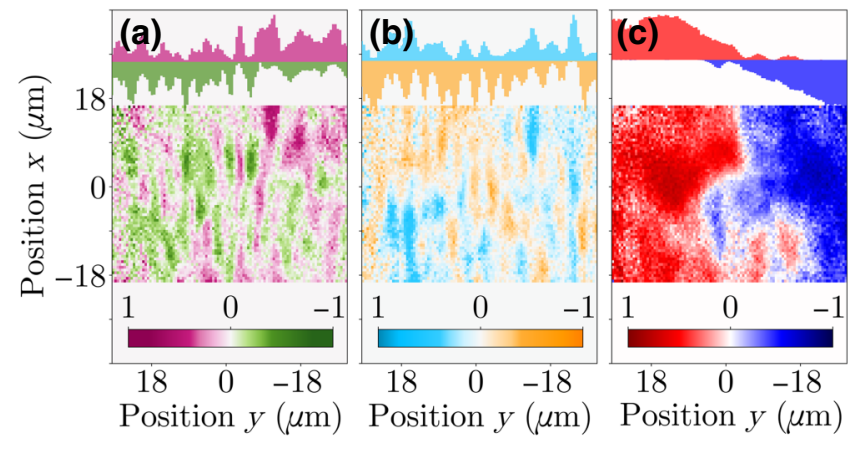

FIG. 5. The persistent spin-helix laser: the Stokes parameters (a) $S_{1}$, (b) $S_{2}$, and (c) $S_{3}$ for real-space imaging. The histograms in the insets at the top correspond to the positive and negative values along the $y$ direction.

Figure 5 shows a real-space image of the Stokes parameters $S_{1}, S_{2}$, and $S_{3}$ [Figs. 5(a)-5(c), respectively]. The maps of the $S_{1}(\vec{r})$ and $S_{2}(\vec{r})$ parameters show the pattern of longitudinal stripes along the $x$ axis, characteristic of the PSH pattern. This proves that spin coherence is occurring in the system, as such a texture is characteristic of a linearly polarized state in the RD coupling regime [19]. To better visualize the PSH pattern, the insets at the top of Fig. 5 show histograms made for the positive and negative Stokes-parameter values along the $y$ axis. We note that the maxima of the histogram for the horizontal polarization $(\mathrm{H})$ coincide with the minima of the histogram corresponding to the vertical polarization (V) Fig. 5(a). The helical polarization pattern is further confirmed by comparison of diagonal and antidiagonal polarizations Fig. 5(b) - actually, according to Eq. (2), every measurement of two orthogonal linear polarizations projects the order of the polarization (spin) helix and will reveal similar stripes.

Contrary to Eq. (2), the map of $S_{3}(\vec{r})$ in Fig. 5(c) demonstrates a distinct spatial separation of the two circular polarizations. The corresponding histogram along the $y$ axis reveals an increasing absolute value of the $S_{3}(\vec{r})$ components with the distance from the excitation spot, where, indeed, $S_{3}(0) \approx 0$. As has been shown in Ref. [19], the spatial distribution of $S_{3}(\vec{r})$, as the consequence of the optical Stern-Gerlach effect, is a useful measure of the amount of inseparability of the orbital and polarization degrees of freedom in space. For $S_{3}^{2}=0$, the system is maximally inseparable, whereas when $S_{3}^{2}=1$, the spin and valley degrees of freedom are completely separable [19,32]. In the center of the excitation spot, the $S_{3}(0)$ component is close to zero and, simultaneously, $S_{1}(\vec{r})$ and $S_{2}(\vec{r})$ both have a periodic dependence given by Eq. (2), as shown in Figs. 5(a) and 5(b). Photons detected far from the excitation spot are mostly circularly polarized - as expected from angular emission $\pm 8^{\circ}$ (or $\pm 1.5 \mu \mathrm{m}^{-1}$ ) and shown in Fig. 4. Thus the polarization and direction degrees of freedom are fully separable and $S_{3}^{2} \approx 1$ [Fig. 5(c)].

\section{CONCLUSIONS}

In summary, we realize a tunable laser based on a liquidcrystal optical microcavity doped with the pyrromethene 580 organic dye. The tunable range reaches $40 \mathrm{~nm}$. The horizontally polarized laser emission is repeatable and can be triggered by single pulses of a pump laser. By transforming the system into the Rashba-Dresselhaus coupling regime, the laser action takes place from the bottoms of two oppositely polarized valleys shifted apart in reciprocal space. Measurements of emissions in real space show the persistent spin-helix lasing, which is a consequence of the spin coherence of the system. The platform that we propose can be used in quantum communication, in which information is encoded through light polarization.

\section{ACKNOWLEDGMENTS}

This work was supported by the National Science Center under Grants No. 2019/35/B/ST3/04147, No. 2019/33/B/ST5/02658, and No. 2017/27/B/ST3/00271, and by the Ministry of National Defense, Republic of Poland, Program-Research Grants Military University of Technology (MUT) 13-995/WAT/2021 and UGB 22840/WAT/2021 for the Laboratory of Crystals Physics and Technology for year 2021 and the European Union Horizon 2020 program, through a Future and Emerging Technologies (FET) Open research and innovation action under Grant Agreement No. 964770 (TopoLight). P.G.L. acknowledges the support of the United Kingdom Engineering and Physical Sciences Research Council (Grant No. EP/M025330/1 on Hybrid Polaritonics), the Russian Foundation for Basic Research (RFBR) under project No. 20-02-00919, and the European Union Horizon 2020 program, through a FET Open research and innovation action under Grant Agreements No. 899141 (PoLLoC) and No. 964770 (TopoLight).

[1] K. J. Vahala, Optical microcavities, Nature 424, 839 (2003).

[2] K. Rechcińska, M. Król, R. Mazur, P. Morawiak, R. Mirek, K. Łempicka, W. Bardyszewski, M. Matuszewski, P. Kula, W. Piecek, P. G. Lagoudakis, B. Piętka, and J. Szczytko, Engineering spin-orbit synthetic Hamiltonians in liquidcrystal optical cavities, Science 366, 727 (2019).

[3] D. Hey and E. Li, Advances in synthetic gauge fields for light through dynamic modulation, R. Soc. Open Sci. 5, 172447 (2018).

[4] L. Lu, J. D. Joannopoulos, and M. Soljačić, Topological photonics, Nat. Photonics 8, 821 (2014).

[5] T. Ozawa, H. M. Price, A. Amo, N. Goldman, M. Hafezi, L. Lu, M. C. Rechtsman, D. Schuster, J. Simon, O. Zilberberg, and I. Carusotto, Topological photonics, Rev. Mod. Phys. 91, 015006 (2019).

[6] V. G. Sala, D. D. Solnyshkov, I. Carusotto, T. Jacqmin, A. Lemaître, H. Terças, A. Nalitov, M. Abbarchi, E. 
Galopin, I. Sagnes, J. Bloch, G. Malpuech, and A. Amo, Spin-Orbit Coupling for Photons and Polaritons in Microstructures, Phys. Rev. X 5, 011034 (2015).

[7] C. E. Whittaker, T. Dowling, A. V. Nalitov, A. V. Yulin, B. Royall, E. Clarke, M. S. Skolnick, I. A. Shelykh, and D. N. Krizhanovskii, Optical analogue of Dresselhaus spinorbit interaction in photonic graphene, Nat. Photonics 15, 193 (2021).

[8] K. Y. Bliokh, F. J. Rodríguez-Fortuño, F. Nori, and A. V. Zayats, Spin-orbit interactions of light, Nat. Photonics 9, 796 (2015).

[9] J. Dalibard, F. Gerbier, G. Juzeliunas, and P. Öhberg, Colloquium: Artificial gauge potentials for neutral atoms, Rev. Mod. Phys. 83, 1523 (2011).

[10] V. Galitski and I. B. Spielman, Spin-orbit coupling in quantum gases, Nature 494, 49 (2013).

[11] C. Weitenberg and J. Simonet, Tailoring quantum gases by Floquet engineering, Nat. Phys. 17, 1342 (2021).

[12] D. N. Basov, R. D. Averitt, and D. Hsieh, Towards properties on demand in quantum materials, Nat. Mater. 16, 1077 (2017).

[13] M. Aidelsburger, S. Nascimbene, and N. Goldman, Artificial gauge fields in materials and engineered systems, C. R. Phys. 19, 394 (2018).

[14] M. S. Rudner and N. H. Lindner, Band structure engineering and non-equilibrium dynamics in Floquet topological insulators, Nat. Rev. Phys. 2, 229 (2020).

[15] K. Lekenta, M. Król, R. Mirek, K. Łempicka, D. Stephan, R. Mazur, P. Morawiak, P. Kula, W. Piecek, P. G. Lagoudakis, B. Piętka, and J. Szczytko, Tunable optical spin Hall effect in a liquid crystal microcavity, Light Sci. Appl. 7, 74 (2018).

[16] A. Manchon, H. C. Koo, J. Nitta, S. M. Frolov, and R. A. Duine, New perspectives for Rashba spin-orbit coupling, Nat. Mater. 14, 871 (2015).

[17] B. A. Bernevig, J. Orenstein, and S.-C. Zhang, Exact SU(2) Symmetry and Persistent Spin Helix in a SpinOrbit Coupled System, Phys. Rev. Lett. 97, 236601 (2006).

[18] J. D. Koralek, C. P. Weber, J. Orenstein, B. A. Bernevig, S.-C. Zhang, S. Mack, and D. D. Awschalom, Emergence of the persistent spin helix in semiconductor quantum wells, Nature 458, 610 (2009).

[19] M. Król, K. Rechcińska, H. Sigurdsson, P. Oliwa, R. Mazur, P. Morawiak, W. Piecek, P. Kula, P. G. Lagoudakis, M. Matuszewski, W. Bardyszewski, B. Pietka, and J. Szczytko, Realizing Optical Persistent Spin Helix and
Stern-Gerlach Deflection in an Anisotropic Liquid Crystal Microcavity, Phys. Rev. Lett. 127, 190401 (2021).

[20] A. J. C. Kuehne and M. C. Gather, Organic lasers: Recent developments on materials, device geometries, and fabrication techniques, Chem. Rev. 116, 12823 (2016).

[21] T. Erdem and H. V. Demir, Colloidal nanocrystals for quality lighting and displays: Milestones and recent developments, Nanophotonics 5, 74 (2016).

[22] Y.-S. Park, J. Roh, B. T. Diroll, R. D. Schaller, and V. I. Klimov, Colloidal quantum dot lasers, Nat. Rev. Mater. 6, 382 (2021).

[23] R. Dąbrowski, P. Kula, and J. Herman, High birefringence liquid crystals, Crystals 3, 443 (2013).

[24] Y. Jiang, Y.-Y. Liu, X. Liu, H. Lin, K. Gao, W.-Y. Lai, and W. Huang, Organic solid-state lasers: A materials view and future development, Chem. Soc. Rev. 49, 5885 (2020).

[25] J. Mysliwiec, A. Szukalska, A. Szukalski, and L. Sznitko, Liquid crystal lasers: The last decade and the future, Nanophotonics 10, 2309 (2021).

[26] F. L. Arbeloa, J. Bañuelos, V. Martínez, T. Arbeloa, and I. L. Arbeloa, Structural, photophysical and lasing properties of pyrromethene dyes, Int. Rev. Phys. Chem. 24, 339 (2005).

[27] C. Mowatt, S. M. Morris, M. H. Song, T. D. Wilkinson, R. H. Friend, and H. J. Coles, Comparison of the performance of photonic band-edge liquid crystal lasers using different dyes as the gain medium, J. Appl. Phys. 107, 043101 (2010).

[28] T. Yagafarov, D. Sannikov, A. Zasedatelev, K. Georgiou, A. Baranikov, O. Kyriienko, I. Shelykh, L. Gai, Z. Shen, D. Lidzey, and P. Lagoudakis, Mechanisms of blueshifts in organic polariton condensates, Commun. Phys. 3, 18 (2020).

[29] Y. Li, X. Ma, X. Zhai, M. Gao, H. Dai, S. Schumacher, and T. Gao, Manipulate polariton condensates by RashbaDresselhaus effect at room temperature, ArXiv:2108.02057.

[30] P. G. Lagoudakis, M. D. Martin, J. J. Baumberg, G. Malpuech, and A. Kavokin, Coexistence of low threshold lasing and strong coupling in microcavities, J. Appl. Phys. 95, 2487 (2004).

[31] W. P. Partridge, N. M. Laurendeau, C. C. Johnson, and R. N. Steppel, Performance of pyrromethene 580 and 597 in a commercial Nd:YAG-pumped dye-laser system, Opt. Lett. 19, 1630 (1994).

[32] N. Korolkova and G. Leuchs, Quantum correlations in separable multi-mode states and in classically entangled light, Rep. Prog. Phys. 82, 056001 (2019). 